\title{
ISOMORPHISMS INDUCED BY AUTOMORPHISMS
}

Dedicated to the memory of Hanna Neumann

JOHN G. THOMPSON

(Received 3 March 1972)

Communicated by M. F. Newman

The object of this note is to record a property of finite, perfect, centrally closed groups, where, by definition, $G$ is centrally closed if and only if whenever $E / Z \cong G$ and $Z \subseteq E^{\prime} \cap Z(E)$, then $Z=1$.

THEOREM. Suppose $G$ is a finite, perfect, centrally closed group and $Z_{1}, Z_{2}$ are central subgroups of $G$ such that $G / Z_{1} \cong G / Z_{2}$. Then $\alpha\left(Z_{1}\right)=Z_{2}$ for some $\alpha \in \operatorname{Aut}(G)$.

Proof. We may assume that $G=F / A$, where $F$ is a free group. Let $Z(G)$ $=R / A$ be the center of $G$. Then $[1$, p. 628 if $]$

$$
R /[R, F]=A /[R, F] \times F^{\prime} \cap R /[R, F] .
$$

Since $G$ is perfect, we have

$$
F=F^{\prime} A, \quad F^{\prime} \cap A=\left(F^{\prime} \cap R\right) \cap A=[F, R],
$$

and so there is an isomorphism

$$
\rho: F / A \cong F^{\prime} /[F, R] .
$$

Let $Z_{i}=L_{i} / A, i=1,2$, so that $L_{i} \subseteq R$. Then

$$
F / L_{1} \cong F|A| L_{1}|A=G| Z_{1} \cong G / Z_{2}=F|A| L_{2} \mid A \cong F / L_{2} .
$$

Thus, as $F$ is free, there is an endomorphism $\pi$ of $F$ which induces an isomorphism of $F / L_{1}$ onto $F / L_{2}$, that is,

$$
\pi\left(L_{1}\right) \subseteq L_{2}, \quad F=\pi(F) \cdot L_{2} .
$$

Hence, $F^{\prime}=\pi(F)^{\prime}\left[\pi(F), L_{2}\right] \cdot L_{2}^{\prime} \subseteq \pi\left(F^{\prime}\right)[F, R] \subseteq F^{\prime}$, and so

$$
F^{\prime}=\pi\left(F^{\prime}\right)[F, R] \text {. }
$$

Also, $Z\left(F / L_{i}\right)=R / L_{i}$, since $G$ is perfect so that its center and second center coincide. Hence, $\pi(R) \subseteq R$, whence $\pi([F, R]) \subseteq[F, R]$, and $\pi$ induces an 
endomorphism $\pi^{*}$ of $F^{\prime} /[F, R]$. By (1), $\pi^{*}$ is onto, and since $F^{\prime} /[F, R]$ is finite, $\pi^{*}$ is an automorphism. Then the composition

$$
F / A \stackrel{\rho}{\longrightarrow} F^{\prime} /[F, R] \stackrel{\pi^{*}}{\longrightarrow} F^{\prime} /[F, R] \stackrel{\rho^{-1}}{\longrightarrow} F / A
$$

is an automorphism of $G$ which carries $Z_{1}$ to $Z_{2}$.

REMARK. If $G$ is a covering group of a simple group $S$, we can use the theorem to help determine all the isomorphism classes of perfect groups $\tilde{G}$ such that $\tilde{G} / Z(\widetilde{G}) \cong S$; we are led to the study of the orbits of Aut $(S)$ on the subgroups of the Schur multiplicator of $S$.

\section{Bibliography}

[1] B. Huppert, Endliche Gruppen, (Springer, 1967).

University of Cambridge

England 